\title{
Thyroid Gland Follicular Lymphoma
}

National Cancer Institute

\section{Source}

National Cancer Institute. Thyroid Gland Follicular Lymphoma. NCI Thesaurus. Code C156410.

A rare follicular lymphoma primarily involving the thyroid gland. 